\title{
EMBEDDING THEOREMS INTO LIPSCHITZ AND BMO SPACES AND APPLICATIONS TO QUASILINEAR SUBELLIPTIC DIFFERENTIAL EQUATIONS
}

\author{
GUOZHEN Lu ${ }^{1}$
}

\begin{abstract}
This paper proves Harnack's inequality for solutions to a class of quasilinear subelliptic differential equations. The proof relies on various embedding theorems into nonisotropic Lipschitz and BMO spaces associated with the vector fields $X_{1}, \ldots, X_{m}$ satisfying Hörmander's condition. The nonlinear subelliptic equations under study include the important p-sub-Laplacian equation, e.g.,$$
\begin{array}{r}
\sum_{j=1}^{m} X_{j}^{*}\left(|X u|^{p-2} X_{j} u\right)=A|X u|^{p}+B|X u|^{p-1}+C|u|^{p-1}+D, \\
1<p<\infty
\end{array}
$$

where $|X u|=\sum_{j=1}^{m}\left(\left|X_{j} u\right|^{2}\right)^{\frac{1}{2}}$ and $A$ is a constant; $B, C$ and $D$ can be in appropriate function spaces. We note that $A$ can be nonzero.
\end{abstract}

\section{Introduction}

One of the main purposes of this paper is to show various embedding theorems into nonisotropic Lipschitz and BMO spaces associated with the vector fields satisfying Hörmander's condition. The other, more importantly, is to apply some of our new theorems proved here to study the local regularity of certain classes of nonlinear subelliptic PDE formed by vector fields. These nonlinear subelliptic equations studied here include the important $p$-sub-Laplacian as a special case.

${ }^{1}$ Results of this paper were presented at the 890th AMS special session at Lexington, Kentucky in March, 1994. The author is supported in part by the National Science Foundation grant \#DMS-9315963. 
Let $\Omega$ be a bounded, open and pathconnected domain in $R^{n}$, and let $X_{1}, \ldots, X_{m}$ be a collection of $C^{\infty}$ real vector fields defined in a neighbourhood of the closure $\bar{\Omega}$ of $\Omega$. For a multi-index $\alpha=\left(i_{1}, \ldots, i_{k}\right)$, denote by $X_{\alpha}$ the commutator $\left[X_{i_{1}},\left[X_{i_{2}}, \ldots,\left[X_{i_{k-1}}, X_{i_{k}}\right]\right], \ldots,\right]$ of length $k=|\alpha|$. Throughout this paper we assume that the vector fields satisfy Hörmander's condition: there exists some positive integer $s$ such that $\left\{X_{\alpha}\right\}_{|\alpha| \leq s}$ span the tangent space of $R^{d}$ at each point of $\Omega$. We can define a metric as follows: An admissible path $\gamma$ is a Lipschitz curve $\gamma:[a, b] \rightarrow \Omega$ such that there exist functions $c_{i}(t), a \leq t \leq b$, satisfying $\sum_{i=1}^{m} c_{i}(t)^{2} \leq 1$ and $\gamma^{\prime}(t)=\sum_{i=1}^{m} c_{i}(t) X_{i}(\gamma(t))$ for almost every $t \in[a, b]$. Then a natural metric on $\Omega$ associated to $X_{1}, \ldots, X_{m}$ is defined by

$$
\begin{array}{r}
\varrho(\xi, \eta)=\min \{b \geq 0: \exists \text { an admissible path } \gamma:[0, b] \rightarrow \Omega \\
\text { such that } \gamma(0)=\xi \text {, and } \gamma(b)=\eta\} .
\end{array}
$$

The metric ball is defined by $B(\xi, r)=\{\eta: \varrho(\xi, \eta)<r\}$. This metric is equivalent to the various other metrics defined in the work of NagelStein-Wainger [NSW]. Note that the Lebesgue measure is doubling with respect to the metric balls as shown in $[\mathbf{N S W}]$. Thus $(\Omega, \varrho)$ is a homogeneous space.

By the Rothschild-Stein lifting theorem (see $[\mathbf{R o S}]$ ), the vector fields $\left\{X_{i}\right\}_{i=1}^{m}$ on $\Omega \subset R^{d}$ can be lifted to vector fields $\left\{\tilde{X}_{i}\right\}_{i=1}^{m}$ in $\tilde{\Omega}=\Omega \times$ $T \subset R^{d} \times R^{N-d}$, where $T$ is the unit ball in $R^{N-d}$ by adding extra variables so that the resulting vector fields are free, i.e., the only linear relation between the commutators of order less than or equal to $s$ at each point of $\tilde{\Omega}$ are the antisymmetric and Jacobi's identity. Let $\mathcal{G}(m, s)$ be the free Lie algebra of steps with $m$ generators, that is the quotient of the free Lie algebra with $m$ generators by the ideal generated by the commutators of order at least $s+1$. Then $\left\{X_{\alpha}\right\}_{|\alpha| \leq s}$ are free if and only if $d=\operatorname{dim} \mathcal{G}(m, s)$. We also define $Q=\sum_{j=1}^{s} j m_{j}$ where $m_{j}$ is the number of linearly independent commutators of length $j$. This integer $Q$ is called the homogeneous dimension associated with the vector fields.

We now define the Sobolev space $W^{1, p}(\Omega)$ to be the completion of $C^{\infty}(\Omega)$ under the norm

$$
\|f\|_{W^{1, p}(\Omega)}=\left(\int_{\Omega}|f|^{p}\right)^{1 / p}+\left(\int_{\Omega}|X f|^{p}\right)^{1 / p},
$$

where $|X f|$ expresses $\left(\sum_{i=1}^{m}\left|X_{i} f\right|^{2}\right)^{\frac{1}{2}}$. We also define $W_{0}^{1, p}(\Omega)$ as the completion of $C_{0}^{\infty}(\Omega)$ under the above norm $\|\cdot\|_{W^{1, p}(\Omega)}$.

Let us review briefly the known results on embedding theorems, especially Poincaré type inequality for vector fields satisfying Hörmander's 
condition. We refer the interested reader to, e.g., [CDG1], [FGW] and $[\mathbf{L} 1]$, for the embedding theorems of Sobolev type (i.e., the functions under consideration are assumed to be with compact support). For embedding theorems on groups, we refer the reader to $[\mathbf{F S}],[\mathbf{K r a}],[\mathbf{V a}]$ and [VS-CC]. For nonsmooth vector fields, extensive study has been given in $[\mathbf{F r}],[\mathbf{F r L}],[\mathbf{F r S}]$ and $[\mathbf{F G u W}]$.

Theorem. Let $E \subset \subset \Omega, 1 \leq p<\infty$, then there exist some $q=$ $q(p) \geq p$ and constants $r_{0}>0, C>0, c \geq 1$, such that for any metric balls $B=B(x, r)$ with $c B=B(x, c r) \subset \Omega, x \in E$, and any $f \in \operatorname{Lip}_{1}(\bar{B})$, the following inequality holds

$$
\left(\frac{1}{|B|} \int_{B}\left|f-f_{B}\right|^{q}\right)^{1 / q} \leq C r\left(\frac{1}{|B|} \int_{B} \sum_{i=1}^{m}\left|X_{i} f\right|^{p}\right)^{1 / p}
$$

provided $0<r<r_{0}$, where $C, c, r_{0}$ depend only on $E, \Omega, f_{B}$ may be taken to be $\frac{1}{|B|} \int_{B} f$.

Such an inequality was first proved by D. Jerison [Jer] for all $1 \leq p<$ $\infty$ and $q=p$. The same inequality in the setting of subelliptic operators was proved by Jerison and Sanchez-Calle in [JeS]. After the work of $[\mathbf{J e r}]$ and $[\mathbf{J e S}]$, the author of the present paper improved the result in $[\mathbf{J}]$ for $p>1$ and extend it to weighted case $([\mathbf{L} 1]-[\mathbf{L 2}])$. Especially, when $1<p<Q$, it is shown in $[\mathbf{L 1}]$ and $[\mathbf{L 2}]$ that $q$ can be taken as $1 \leq q \leq \frac{Q p}{Q-p}$.

We remark here that by the Rellich-Kondrachov compact embedding theorem for vector fields satisfying Hörmander's condition (see, e.g., [L4]) and together with a well-known compactness argument (see, e.g., $[\mathbf{L 5}])$, one can recapture the proof of the Poincaré inequality with $2 B$ on the right side for all $1 \leq q<\frac{p Q}{Q-p}$ except the endpoint $q=\frac{Q p}{Q-p}$. However, such a Poincaré inequality usually involves a constant $C$ possibly depending on the ball $B$ in general.

When $p=Q$, the following inequality was shown in $[\mathbf{L 3}]$ that for all balls $B$ with $c B \subset \Omega$ :

$$
\frac{1}{|B|} \int_{B} \exp \left(A\left(\frac{\left|f-f_{B}\right|}{\left\|\sum_{i=1}^{m} \mid X_{i} f\right\|_{L^{p}(B)}}\right)^{\frac{Q}{Q-1}}\right) d x \leq C
$$

where $A>0, C>0$ and $c \geq 1$ are absolute constants provided that $f \in \operatorname{Lip}_{1}(\bar{B})$ is not constant.

All the Poincaré type inequalities proved so far are with the restriction $\frac{1}{p}-\frac{1}{q} \leq \frac{1}{Q}$. However, if we consider embedding theorems on the 
Campanato-Morrey spaces, we will get inequalities with larger differences $\frac{1}{p}-\frac{1}{q}$. To state the theorems proved in [L3], we briefly define the Campanato-Morrey spaces as follows:

Let now $f_{B}=\frac{1}{|B|} \int_{B} f(y) d y$ be the average over the ball $B$ of the function $f$. We define the following two types of Campanato-Morrey norms:

Fix any $R>0$. Let $\mathcal{L}^{p, \lambda}(\Omega)$ be the spaces of all functions $f \in L_{\text {loc }}^{p}(\Omega)$ such that

$$
\|f\|_{\mathcal{L}^{p, \lambda}(\Omega)}=\sup _{B}\left(\rho(B)^{\lambda}|B|^{-1} \int_{B}\left|f-f_{B}\right|^{p}\right)^{\frac{1}{p}}<\infty
$$

where the sup is taken over all the balls $B=B(x, r)$ with $c B=B(x, c r) \subset$ $\Omega$ with $x \in E \subset \subset \Omega$ for some subset $K$ and $\rho(B)=r$ (the radius of the ball $B) \leq R$. It is easy to see that two elements of $\mathcal{L}^{p, \lambda}$ can be identified if they only differ by a constant.

We also define the space $M^{p, \lambda}$ of functions $f \in L_{\text {loc }}^{p}(\Omega)$ such that

$$
\|f\|_{M^{p, \lambda}(\Omega)}=\sup _{B}\left(\rho(B)^{\lambda}|B|^{-1} \int_{B}|f|^{p}\right)^{\frac{1}{p}}<\infty,
$$

where the sup is taken in the same sense as above.

Then one of the main theorems proved in [L3] is the following:

Theorem. Given any $f \in W_{\mathrm{loc}}^{1, p}(\Omega)$ the following is true:

$$
\|f\|_{\mathcal{L}^{p^{*}, \lambda}(\Omega)} \leq C\left\|\sum_{i=1}^{m}\left|X_{i} f\right|\right\|_{M^{p, \lambda}(\Omega)}
$$

where $0<\lambda \leq Q, 1<p<\lambda$ and $p^{*}=\frac{\lambda p}{\lambda-p}$, provided that the number $R>0$ is small enough in the definition of the spaces $\mathcal{L}^{p, \lambda}(\Omega)$ and $M^{p, \lambda}(\Omega)$.

We note in the above that $\frac{1}{p}-\frac{1}{p^{*}}=\frac{1}{\lambda}$ can be taken much larger than the known gap in the Poincaré inequality, which is known to be true so far for $\frac{1}{Q}$.

Recently, Franchi, Wheeden and the author showed in $[$ FLW] that a Poincaré inequality holds when $p=1$ and $q=\frac{Q}{Q-1}$ (when $p=q=1$, the result was due to Jerison $[\mathbf{J e r}])$. We mention that this endpoint result for $p=1$ contains certain important geometric information. Indeed, applying this Poincaré inequality, we also derived a relative isoperimetric 
inequality $([\mathbf{F L W}])$. A new representation formula was derived in $[\mathbf{F L W}]$ which improves the one obtained in $[\mathbf{L 1}]$. Results in $[\mathbf{F L W}]$ also sharpen the exponents given in those inequalities in $[\mathbf{L} \mathbf{1}]-[\mathbf{L} 2]$.

One of the main goals of this paper is to show some new embedding theorems for Hörmander's vector fields which will complement the theorems mentioned above. The current theorems shown here together with the previously known ones will give a fairly complete picture of embedding theorems for vector fields of Hörmander's type. More importantly, we will employ these new theorems to prove a Harnack inequality for a certain class of quasilinear subelliptic differential equations formed by vector fields satisfying Hörmander's condition.

We first state the embedding theorems. From now on, we use frequently $|X f|$ to express $\left(\sum_{i=1}^{m}\left|X_{i} f\right|^{2}\right)^{\frac{1}{2}}$.

Theorem 1.1. Suppose $p>Q$. Then there exists some constant $c \geq 1$ such that for any $f \in W^{1, p}(\Omega)$, for any ball $B_{R}$ with $c B_{R} \subset \Omega$ we have

$$
\sup _{x, y \in B_{R}}|f(x)-f(y)| \leq C\left|B_{R}\right|^{\frac{1}{Q}-\frac{1}{p}}\|X f\|_{L^{p}\left(c B_{R}\right)} .
$$

Furthermore $f \in C_{\mathrm{loc}}^{0, \gamma}(\Omega)$ (the local nonisotropic Lipschitz space), where $\gamma=1-\frac{Q}{p}$, in the sense that for any compact subset $K \subset \Omega$

$$
\sup _{x, y \in K, x \neq y} \frac{|f(x)-f(y)|}{\rho(x, y)^{\gamma}} \leq C\|X f\|_{L^{p}(\Omega)},
$$

provided that one of the metric balls $B(x, \varrho(x, y))$ and $B(y, \varrho(y, x))$ is contained in $\Omega$.

The embedding $W^{1, p}(\Omega) \rightarrow C_{\mathrm{loc}}^{0, \beta}(\Omega)$ is compact provided $\beta<\gamma$.

Remark. If we assume $f \in W_{0}^{1, p}(\Omega), p>Q$, then we can show $f \in C^{0, \gamma}(\bar{\Omega})$, i.e.,

$$
\sup _{x, y \in \Omega, x \neq y} \frac{|f(x)-f(y)|}{\rho(x, y)^{\gamma}} \leq C\|X f\|_{L^{p}(\Omega)} .
$$

Theorem 1.2. Given any $1 \leq p<\infty$ and $c \geq 1$. Suppose $K$ and $0<\alpha \leq 1$ are two positive constants. Let $f \in W^{1, p}(\Omega)$ satisfy

$$
\int_{B_{R}}|X f|^{p}(x) d x \leq K^{p}\left|B_{R}\right| R^{(-1+\alpha) p},
$$


for all balls $B_{R} \subset \Omega$, then $f \in C_{\text {loc }}^{0, \alpha}(\Omega)$ and for any ball $B_{R}$ with $c B_{R} \subset \Omega$ we have

$$
\sup _{x, y \in B_{R}}|f(x)-f(y)| \leq C K R^{\alpha}
$$

where $C=C(Q, \alpha)$. Moreover, for any compact subset $K \subset \Omega$ there exists $r_{0}>0$, we have

$$
\sup _{x, y \in K, x \neq y, \varrho(x, y) \leq r_{0}} \frac{|f(x)-f(y)|}{\rho(x, y)^{\alpha}} \leq C K .
$$

Theorem 1.3. Given any $1 \leq p<\infty$ and $c \geq 1$. Suppose $f \in$ $W^{1, p}(\Omega)$ and also that there exists a positive constant $K$ such that

$$
\int_{B_{R}}|X f|^{p}(x) d x \leq K^{p}\left|B_{R}\right| R^{-p}
$$

for all balls $B_{R} \subset \Omega$. Then there exist positive constants $\sigma$ and $C$ such that for all balls $B_{R}$ with $c B_{R} \subset \Omega$

$$
\int_{B_{R}} \exp \left(\frac{\sigma}{K}\left|f-f_{B}\right|\right)(x) d x \leq C\left|B_{R}\right| .
$$

We remark here that Theorems (1.2) and (1.3) do not involve the homogeneous dimension $Q$, both the theorems and proofs work in more general settings, say, for Grushin or nonsmooth vector fields (see $[\mathbf{F G u W}]$ ).

By employing the above theorems when $1<p<\infty$, we shall establish certain Harnack inequalities for weak solutions, subsolutions, and supersolutions of quasilinear second order subelliptic partial differential equations of the form

$\sum_{j=1}^{(1.4)} X_{j}^{*} A_{j}\left(x, u, X_{1} u, X_{2} u, \ldots, X_{m} u\right)+B\left(x, u, X_{1} u, X_{2} u, \ldots, X_{m} u\right)=0$

where $X_{j}^{*}$ is the adjoint of $X_{j}$, which is not necessarily a vector field in general; $u(x)$ is assumed to be in $W_{\text {loc }}^{1, p}(\Omega)$. As a special case of our theorems, we will be able to obtain the local regularity for the well-known sub-Laplacian.

The Harnack inequality will be established under certain structural assumptions on the equation (1.4) (see Theorems (3.9), (3.13), (3.15) and Corollary (3.11) in Section 3).

We now let $x=\left(x_{1}, \ldots, x_{n}\right), \eta=\left(\eta_{1}, \ldots, \eta_{m}\right)$ denote vectors in $R^{n}$ and $R^{m}$ respectively and $X u=\left(X_{1} u, \ldots, X_{m} u\right)$. Let $A(x, u, \eta)=$ 
$\left(A_{1}(x, u, \eta), \ldots, A_{m}(x, u, \eta)\right)$ and $B(x, u, \eta)$ be, respectively, vector and scalar measurable functions defined on $\Omega \times R \times R^{m}$, where $\Omega$ is a domain in $R^{n}$.

The structure of the equation (1.4) throughout this paper will be assumed to satisfy the following:

$$
\begin{aligned}
|A(x, u, \eta)| & \leq a_{0}|\eta|^{p-1}+\left(a_{1}(x)|u|\right)^{p-1}+\left(a_{3}(x)\right)^{p-1} \\
\eta \cdot A(x, u, \eta) & \geq|\eta|^{p}-\left(a_{2}(x)|u|\right)^{p}-\left(a_{4}(x)\right)^{p} \\
|B(x, u, \eta)| & \leq b_{0}|\eta|^{p}+b_{1}(x)|\eta|^{p-1}+\left(b_{2}(x)\right)^{p}|u|^{p-1}+\left(b_{3}(x)\right)^{p}
\end{aligned}
$$

where $1<p<\infty, a_{0}, b_{0}$ are constants, $a_{i}(x), b_{i}(x)$ are nonnegative measurable functions satisfying certain integrability properties which will be described in Section 3.

Such type of equations when $X_{i}=\frac{\partial}{\partial x_{i}}(i=1,2, \ldots, n)$ in $R^{n}$ have been studied in the literature (see $[\mathbf{S e r}],[\mathbf{G i T}],[\mathbf{T r u}],[\mathbf{Z i e}]$ ). We point out here that the equation (1.4) has been studied in [CDG1] when $p$ is restricted to $1<p \leq Q$ under the assumption of $b_{0}=0$. Our theorems proved in this paper include all $1<p<\infty$ and also $b_{0} \neq 0$. Moreover, the results in $[\mathbf{C D G 1}$ require higher integrability conditions on the coefficients $a_{i}(x)(i=1,2,3,4), b_{j}(x)(j=1,2,3)$ than the ones given here (see Section 3 for details). For example, by our theorems in Section 3 the solutions of, e.g., the following very simple equation for all $1<p<\infty$ satisfies a uniform Harnack inequality:

$$
\sum_{j=1}^{m} X_{j}^{*}\left(|X u|^{p-2} X_{j} u\right)=A|X u|^{p}+B|X u|^{p-1}+C|u|^{p-1}+D
$$

where again $|X u|=\sum_{j=1}^{m}\left(\left|X_{j} u\right|^{2}\right)^{\frac{1}{2}}, A$ is a constant; $B, C$ and $D$ are in appropriate function spaces which will be specified below. We should also mention that when $1<p \leq Q$ we shall assume the solutions are a priori bounded (when $b_{0}=0$ such an assumption can be dropped, see Section 3 ) while when $p>Q$ the local boundedness and Hölder continuity of the solutions follows by the embedding Theorem (1.1) proved in this paper without obtaining Harnack inequality first. However, one still needs to prove the Harnack inequality for $p>Q$ because Hölder continuity of the solutions does not lead to this.

We also remark that the proofs of the Harnack inequalities for the solutions of the equation (1.4) rely on Sobolev embedding theorems (see for example $[\mathbf{L 1}],[\mathbf{F G W}]$ ) and embedding theorems into Lipschitz and BMO spaces proved here. We will also need to adapt the well-known Moser's iteration argument [Mos] to our nonlinear subelliptic case. For 
elliptic Euclidean case, we refer the interested reader to $[\mathbf{L a U}]$, [Mos], $[\mathbf{N a s}]$, [Ser], [GiT], [Tru], [Zie] and references therein. Our Harnack inequalities extend to the subelliptic context results due to J. Serrin, N. Trudinger, Ladyzhenskaya and Ural'tseva (see [Ser], [Tru], [LaU]). We also mention that subelliptic variational problems have been studied by $\mathrm{Xu}$ in $[\mathbf{X} \mathbf{1}]$.

The organization of the paper is as follows: Section 2 contains the proofs of the embedding theorems which will be needed in proving the Harnack inequality. Section 3 devotes the proof of the Harnack inequality, Hölder continuity, and estimates of the solutions at the boundary.

We will use the letters $C, c$, etc., to denote the absolute constants and may differ from line to line.

\section{Proofs of Theorems (1.1), (1.2) and (1.3)}

We recall again that by the Rothschild-Stein lifting theorem (see $[\mathbf{R o S}])$ the vector fields $\left\{X_{i}\right\}_{i=1}^{m}$ on $\Omega \subset R^{d}$ can be lifted to vector fields $\left\{\tilde{X}_{i}\right\}_{i=1}^{m}$ in $\tilde{\Omega}=\Omega \times T \subset R^{d} \times R^{N-d}$, where $T$ is the unit ball in $R^{N-d}$.

There is also a metric $\tilde{\varrho}: \tilde{\Omega} \times \tilde{\Omega} \rightarrow R^{+}$associated with the lifted vector fields $\tilde{X}_{1}, \ldots, \tilde{X_{m}}$. We note that the Lebesgue measure of the ball $|\tilde{B}(\xi, r)| \approx r^{Q}$, where $Q$ is the homogeneous dimension of $G$, and $\tilde{B}(\xi, r)$ is the metric ball in $(\tilde{\Omega}, \tilde{\varrho})$. Thus $(\tilde{\Omega}, \tilde{\varrho})$ is a homogeneous space in the sense of Coifman and Weiss. We should mention the proofs given in this section are not the simpliest ones.

The following lemma is necessary in order to show Theorem (1.1).

Lemma 2.1. Given any metric ball $\tilde{B} \subset \tilde{\Omega}$ and any Lipschitz continuous function $\tilde{f} \in \operatorname{Lip}_{1}(\tilde{\Omega})$. Then there exist constants $c \geq 1$ and $C \geq 1$ such that for any $\xi \in \tilde{B}$ and any constant $C_{0}$ the following is true:

$$
\left|\tilde{f}(\xi)-\tilde{f}_{\tilde{B}}\right| \leq C \int_{c \tilde{B}} \frac{M\left(\left(\sum_{i=1}^{m}\left|\tilde{X}_{i} \tilde{f}\right|+\left|\tilde{f}-C_{0}\right|\right) \chi_{c \tilde{B}}\right)(\eta)}{\tilde{\varrho}(\xi, \eta)^{Q-1}} d \eta
$$

where $\tilde{f}_{\tilde{B}}=\frac{1}{|\tilde{B}|} \int_{\tilde{B}} \tilde{f}(\eta) d \eta$, and $\tilde{\varrho}(\xi, \eta)$ is the metric distance associated to the lifted vector fields $\left\{\tilde{X}_{i}\right\}_{i=1}^{m} ; M(g)$ is the Hardy-Littlewood maximal function for $g$.

This lemma was essentially proved in $[\mathbf{L} \mathbf{1}]$ (Lemma (3.2) in [L1]). In $[\mathbf{L 1}]$ it was shown that there is a constant $C_{\tilde{B}}$ such that

$$
\left|\tilde{f}(\xi)-C_{\tilde{B}}\right| \leq \int_{c \tilde{B}} \frac{M\left(\left(\sum_{i=1}^{m}\left|\tilde{X}_{i} \tilde{f}\right|+|\tilde{f}|\right) \chi_{c \tilde{B}}\right)(\eta)}{\tilde{\varrho}(\xi, \eta)^{Q-1}} d \eta .
$$


But we can show such a constant $C_{\tilde{B}}$ can be replaced by $\tilde{f}_{\tilde{B}}$. Moreover, if we replace the function $\tilde{f}$ by $\tilde{f}-C_{0}$, we will get Lemma (2.1). Actually in the proof below we will take $C_{0}=\tilde{f}_{c} \tilde{B}$.

Remark. In the above representation formula, it contains the HardyLittlewood maximal function and also the zero order term $\left|\tilde{f}-C_{0}\right|$. Such a formula is good enough for most $L^{p}$ estimates for $p>1$ as demonstrated in $[\mathbf{L 1}]-[\mathbf{L 3}]$. The proof of Theorem (1.1) given below by using this formula is interesting itself when we get rid of the Maximal function by using the boundedness of the maximal function in $L^{p}$ norm and control the terms containing the zero-order term $\left|\tilde{f}-C_{0}\right|$ by using the known Poincaré inequality from $L^{p}$ to $L^{p}$. (See the similar argument in [L3].) Of course, the proof can be much simplified by using the new representation formula obtained in $[\mathbf{F L W}]$. We thought the proof of Theorem (1.1) given below may have its own interest.

Before we start to prove the Theorems (1.1), (1.2) and (1.3), we briefly explain how the proofs go. We will first prove the theorems for free vector fields $\left\{\tilde{X}_{i}\right\}$ and the functions $\tilde{f}$ defined on $\tilde{\Omega}$. Secondly, for any function $f$ defined on $\Omega$ which satisfies the assumptions in the theorems associated with the vector fields $\left\{X_{i}\right\}$, we define the new function $\tilde{f}(\xi)=\tilde{f}(x, t)=$ $f(x)$ for $x \in \Omega$ and $\xi \in \tilde{\Omega}$ and we prove for so defined $\tilde{f}$ it satisfies the conditions associated with the lifted vector fields $\left\{\tilde{X}_{i}\right\}$. Thirdly, we then show the conclusions of the theorems for so defined $\tilde{f}$ will lead to the conclusions for the original function $f$.

We also mention that on the nilpotent Lie group some similar results to our Theorem (1.1) were derived in $[\mathbf{F o l}],[\mathbf{S C}],[\mathbf{C o u}]$ and $[\mathbf{K r a}]$.

Proof of Theorem (1.1): We first show that the theorem holds for $\tilde{f} \in \operatorname{Lip}_{1}(\tilde{\Omega})$. The general case follows by an argument of approximation. Given any ball $\tilde{B} \subset \tilde{\Omega}$, and any $\tilde{f} \in W^{1, p}(\tilde{\Omega}) \cap \operatorname{Lip}_{1}(\tilde{\Omega}), p>Q$. Let $\tilde{f}_{\tilde{B}}=$ $\frac{1}{|\tilde{B}|} \int_{\tilde{B}} \tilde{f}(\eta) d \eta$. Taking $C_{0}=\tilde{f}_{c \tilde{B}}$ in Lemma (2.1), then by Lemma (2.1), for any $\xi \in \tilde{B}$,

$$
\begin{aligned}
\left|\tilde{f}(\xi)-\tilde{f}_{\tilde{B}}\right| \leq & C \int_{c \tilde{B}} \frac{M\left(\left(\sum_{i=1}^{m}\left|\tilde{X}_{i} \tilde{f}\right|+\left|\tilde{f}-\tilde{f}_{c \tilde{B}}\right|\right) \chi_{c \tilde{B}}\right)(\eta)}{\tilde{\varrho}(\xi, \eta)^{Q-1}} d \eta \\
\leq & C\left(\int_{c \tilde{B}}\left(M\left(\left(\sum_{i=1}^{m}\left|\tilde{X}_{i} \tilde{f}\right|+\left|\tilde{f}-\tilde{f}_{c \tilde{B}}\right|\right) \chi_{c \tilde{B}}\right)(\eta)\right)^{p} d \eta\right)^{1 / p} \\
& \cdot\left(\int_{c \tilde{B}} \frac{1}{\tilde{\varrho}(\xi, \eta)^{(Q-1) p^{\prime}}} d \eta\right)^{1 / p^{\prime}}
\end{aligned}
$$




$$
\begin{aligned}
& \leq C\left(\int_{c \tilde{B}}\left(\left(\sum_{i=1}^{m}\left|\tilde{X}_{i} \tilde{f}\right|+\left|\tilde{f}-\tilde{f}_{c \tilde{B}}\right|\right)(\eta)\right)^{p} d \eta\right)^{1 / p} \cdot \rho(\tilde{B})^{1-\frac{Q}{p}} \\
& \leq C(\rho(\tilde{B})+1)\left(\int_{c \tilde{B}} \sum_{i=1}^{m}\left|\tilde{X}_{i} \tilde{f}\right|^{p}\right)^{1 / p} \rho(\tilde{B})^{1-\frac{Q}{p}} .
\end{aligned}
$$

In the above we have used the Hölder's inequality in the second inequality, the $L^{p}$ boundedness of the Hardy-Littlewood maximal function in the third inequality and also the Poincaré inequality in the fourth inequality. So

$$
\sup _{\xi \in \tilde{B}}\left|\tilde{f}(\xi)-\tilde{f}_{\tilde{B}}\right| \leq C \rho(\tilde{B})^{1-\frac{Q}{p}}(\rho(\tilde{B})+1)|| \tilde{X} \tilde{f} \|_{L^{p}(c \tilde{B})} .
$$

Then for $\xi, \eta \in \tilde{B}$,

$$
\begin{aligned}
|\tilde{f}(\xi)-\tilde{f}(\eta)| \leq\left|\tilde{f}(\xi)-\tilde{f}_{\tilde{B}}\right|+\left|\tilde{f}(\eta)-\tilde{f}_{\tilde{B}}\right| & \\
& \leq C \rho(\tilde{B})^{1-\frac{Q}{p}}(\rho(\tilde{B})+1) \|\left.\tilde{X} \tilde{f}\right|_{L^{p}(c \tilde{B})} .
\end{aligned}
$$

Now let $\xi, \eta \in \tilde{\Omega}$, and set $\gamma=1-\frac{Q}{p}$ and $\tilde{B}=\tilde{B}(\xi, \tilde{\varrho}(\xi, \eta))$. Then

$$
\begin{aligned}
|\tilde{f}(\xi)-\tilde{f}(\eta)| \leq C \tilde{\varrho}(\xi, \eta)^{\gamma}(\tilde{\varrho}(\xi, \eta)+1)\|\tilde{X} \tilde{f}\|_{L^{p}(c \tilde{B})} & \\
& \leq C(\Omega) \tilde{\varrho}(\xi, \eta)^{\gamma}\|\tilde{X} \tilde{f}\|_{L^{p}(c \tilde{B})} .
\end{aligned}
$$

Now given any function $f \in W^{1, p}(\Omega)$ and $p>Q$, and any metric ball $B=B\left(x_{0}, r\right) \subset \Omega$, and any $x, y \in B$, we can define the ball $\tilde{B}=\tilde{B}\left(\left(x_{0}, 0\right), r\right) \subset \tilde{\Omega}$. Then $\xi=(x, 0)$ and $\eta=(y, 0) \in c \tilde{B}$. If we set $\tilde{f}(\xi)=f(z)$ for all $\xi=(z, t) \in \tilde{\Omega}, z \in \Omega$, then by the above formula

$$
\begin{aligned}
|f(x)-f(y)|=|\tilde{f}(\xi)-\tilde{f}(\eta)| \leq C(\tilde{\Omega}) \tilde{\varrho}(\xi, \eta)^{\gamma}\|\tilde{X} \tilde{f}\|_{L^{p}(c \tilde{B})} \\
\leq C(\Omega) \rho(B)^{\gamma}\|X f\|_{L^{p}(c B)}
\end{aligned}
$$

by noticing $\tilde{X}_{i} \tilde{f}(\xi)=X_{i} f(z)$ and $\|\tilde{X} \tilde{f}\|_{L^{p}(c \tilde{B})} \leq C(\Omega)\|X f\|_{L^{p}(c B)}$.

We now consider any $x, y \in \Omega$. If we assume that either $B=$ $B(x, \rho(x, y))$ or $B=B(y, \rho(x, y))$ is contained in $\Omega$, we then get

$$
|f(x)-f(y)| \leq C \varrho(x, y)^{\gamma}\|X f\|_{L^{p}(c B)} \leq C \varrho(x, y)^{\gamma}\|X f\|_{L^{p}(\Omega)} .
$$

Therefore, the assertions in Theorem (1.1) follow.

The compact embedding follows easily from the Ascoli-Arzela Theorem. We omit the details here.

We now turn to the proof of Theorem (1.2). We first state the following lemma: 
Lemma 2.2. Given any metric ball $\tilde{B}=\tilde{B}\left(\xi_{0}, r\right) \subset \tilde{\Omega}$ and any Lipschitz continuous function $\tilde{f} \in \operatorname{Lip}_{1}(\tilde{\Omega})$. Then there exist constants $c \geq 1$ and $C \geq 1$ such that for any $\xi \in \tilde{B}$ the following is true:

$$
\left|\tilde{f}(\xi)-\tilde{f}_{\tilde{B}}\right| \leq C \int_{c \tilde{B}} \frac{\sum_{i=1}^{m}\left|\tilde{X}_{i} \tilde{f}\right|(\eta)}{\tilde{\varrho}(\xi, \eta)^{Q-1}} d \eta
$$

where $\tilde{f}_{\tilde{B}}=\frac{1}{|\tilde{B}|} \int_{\tilde{B}} \tilde{f}(\eta) d \eta$, and $\tilde{\varrho}(\xi, \eta)$ is the metric distance associated to the vector fields $\left\{\tilde{X}_{i}\right\}_{i=1}^{m}$.

For general free vector fields of Hörmander type, this lemma is a recent result showed in $[\mathbf{F L W}]$ (even for the original vector fields, the above formula was also proved in [FLW], but we do not need that version here). We also remark here that such a representation formula for functions compactly supported in the ball is immediate by the fundamental solution estimate for the sum of squares (see $[\mathbf{F e S}],[\mathbf{F e P}],[\mathbf{N S W}],[\mathbf{S a n}]$ ).

Since we do not have the adapted "Polar Coordinates" in the setting of Hörmander vector fields, we will prove the theorem by cutting the kernel on metric "annulus".

Proof of Theorem (1.2): We only prove the theorem for the case $p=1$. The general case for $p>1$ follows by the Hölder inequality by observing that

$$
\frac{1}{|B|} \int_{B}|X f| \leq\left(\frac{1}{|B|} \int_{B}|X f|^{p}\right)^{\frac{1}{p}}
$$

Given any metric ball $\tilde{B}=\tilde{B}\left(\xi_{0}, R\right) \subset \tilde{\Omega}$. Note for any $\xi \in \tilde{B}, \tilde{B} \subset$ $\tilde{B}(\xi, c R)$ for some absolute constant $c \geq 1$. Then by Lemma (2.2) for any $\xi \in \tilde{B}$,

$$
\left|\tilde{f}(\xi)-\tilde{f}_{\tilde{B}}\right| \leq C \int_{c \tilde{B}(\xi, c R)} \frac{\sum_{i=1}^{m}\left|\tilde{X}_{i} \tilde{f}\right|(\eta)}{\varrho(\xi, \eta)^{Q-1}} d \eta
$$

Note

$$
\begin{aligned}
\int_{c \tilde{B}(\xi, c R)} & \sum_{i=1}^{m}\left|\tilde{X}_{i} \tilde{f}\right|(\eta) \tilde{\varrho}(\xi, \eta)^{1-Q} d \eta \\
& \leq C \sum_{k=0}^{\infty} \int_{\tilde{B}\left(\xi, c 2^{-k+1} R\right) \backslash \tilde{B}\left(\xi, c 2^{-k} R\right)} \sum_{i=1}^{m}\left|\tilde{X}_{i} \tilde{f}\right|(\eta) \tilde{\varrho}(\xi, \eta)^{1-Q} d \eta \\
& \leq C \sum_{k=0}^{\infty}\left(2^{-k} R\right)^{1-Q} \int_{\tilde{B}\left(\xi, c 2^{-k+1} R\right)} \sum_{i=1}^{m}\left|\tilde{X}_{i} \tilde{f}\right|(\eta) d \eta \\
& \leq C \sum_{k=0}^{\infty}\left(2^{-k} R\right)^{1-Q} \cdot K\left(2^{-k} R\right)^{Q-1+\alpha} \\
& \leq C K R^{\alpha} .
\end{aligned}
$$


Therefore, for any $\xi, \eta \in \tilde{B}$,

$$
|\tilde{f}(\xi)-\tilde{f}(\eta)| \leq C K R^{\alpha},
$$

where $R=\rho(\tilde{B})$.

Reasoning as in the proof of Theorem (1.1), we can actually show

$$
|\tilde{f}(\xi)-\tilde{f}(\eta)| \leq C K \tilde{\varrho}(\xi, \eta)^{\alpha} .
$$

For any given ball $B \subset \Omega$ and function $f \in W^{1, p}(\Omega)$, as in the proof of Theorem (1.1), we define the new function $\tilde{f}(\xi)=\tilde{f}(x)$ for $\xi=(x, t) \in \tilde{\Omega}$, where $x \in \Omega$, and the ball $\tilde{B}$. It is easy to check that the condition for the function $f$

$$
\int_{B_{R}}|X f|^{p}(x) d x \leq K^{p}\left|B_{R}\right| R^{(-1+\alpha) p},
$$

for all balls $B_{R} \subset \Omega$ for the original vector fields will lead to the same condition for the function $\tilde{f}$ defined by $f$

$$
\int_{\tilde{B}_{R}}|\tilde{X} \tilde{f}|^{p}(\xi) d \xi \leq K^{p}\left|\tilde{B}_{R}\right| R^{(-1+\alpha) p},
$$

for all balls $\tilde{B}_{R} \subset \tilde{\Omega}$. Thus arguing as in the proof of Theorem (1.1), we get the desired result.

Proof of Theorem (1.3): There are several ways to derive this lemma. One simplest way is to use the $L^{p}$ to $L^{p}$ Poincaré inequlity, and then use the known John-Nirenberg Theorem. But we will give the proof here without using the known Poincaré inequlity at all.

Again, we will cut the kernel on the metric annuli. Recall for $\xi \in \tilde{B}=$ $\tilde{B}\left(\xi_{0}, R\right) \subset \tilde{\Omega}$ the following holds:

$$
\left|\tilde{f}(\xi)-\tilde{f}_{\tilde{B}}\right| \leq C \int_{c \tilde{B}} \frac{\sum_{i=1}^{m}\left|\tilde{X}_{i} \tilde{f}\right|(\eta)}{\tilde{\varrho}(\xi, \eta)^{Q-1}} d \eta
$$

Note for any given $q \geq 1$ we have,

$$
\begin{aligned}
\int_{c \tilde{B}} \frac{\sum_{i=1}^{m}\left|\tilde{X}_{i} \tilde{f}\right|(\eta)}{\tilde{\varrho}(\xi, \eta)^{Q-1}} d \eta \\
=\int_{c \tilde{B}} \sum_{i=1}^{m}\left|\tilde{X}_{i} \tilde{f}\right|(\eta) \tilde{\varrho}(\xi, \eta)^{\left(\frac{1}{Q q}-1\right) \frac{Q}{q}} \cdot \tilde{\varrho}(\xi, \eta)^{Q\left(1-\frac{1}{q}\right)\left(\frac{1}{Q q}+\frac{1}{Q}-1\right)} d \eta \\
\leq\left(\int_{c \tilde{B}} \sum_{i=1}^{m}\left|\tilde{X}_{i} \tilde{f}\right|(\eta) \tilde{\varrho}(\xi, \eta)^{\left(\frac{1}{Q q}-1\right) Q} d \eta\right)^{1 / q} \\
\\
\quad\left(\int_{c \tilde{B}} \tilde{\varrho}(\xi, \eta)^{Q\left(\frac{1}{Q q}+\frac{1}{Q}-1\right)} \sum_{i=1}^{m}\left|\tilde{X}_{i} \tilde{f}\right|(\eta) d \eta\right)^{\frac{q-1}{q}} .
\end{aligned}
$$


The last inequality above follows by the Hölder inequality.

We also note

$$
\begin{aligned}
\int_{\tilde{B}} \int_{c \tilde{B}} & \sum_{i=1}^{m}\left|\tilde{X}_{i} \tilde{f}\right|(\eta) \tilde{\varrho}(\xi, \eta)^{\left(\frac{1}{Q q}-1\right) Q} d \eta d \xi \\
& \leq C \sup _{\eta \in c \tilde{B}} \int_{c \tilde{B}} \tilde{\varrho}(\xi, \eta)^{\left(\frac{1}{Q q}-1\right) Q} d \xi \cdot\|\tilde{X} \tilde{f}\|_{L^{1}(c \tilde{B})} \\
& \leq C K q R^{1 / q} R^{Q-1} .
\end{aligned}
$$

On the other hand, by noticing $\tilde{B} \subset \tilde{B}(\xi, c R)$ for any $\xi \in \tilde{B}$, we get

$$
\begin{aligned}
\int_{c \tilde{B}} & \sum_{i=1}^{m}\left|\tilde{X}_{i} \tilde{f}\right|(\eta) \tilde{\varrho}(\xi, \eta)^{Q\left(\frac{1}{Q q}+\frac{1}{Q}-1\right)} d \eta \\
& \leq \int_{c \tilde{B}(\xi, c R)} \sum_{i=1}^{m}\left|\tilde{X}_{i} \tilde{f}\right|(\eta) \tilde{\varrho}(\xi, \eta)^{Q\left(\frac{1}{Q q}+\frac{1}{Q}-1\right)} d \eta \\
& \leq C \sum_{k=0}^{\infty} \int_{\tilde{B}\left(\xi, c 2^{-k+1} R\right) \backslash \tilde{B}\left(\xi, c 2^{-k} R\right)} \sum_{i=1}^{m}\left|\tilde{X}_{i} \tilde{f}\right|(\eta) \tilde{\varrho}(\xi, \eta)^{Q\left(\frac{1}{Q q}+\frac{1}{Q}-1\right)} d \eta \\
& \leq C \sum_{k=0}^{\infty}\left(2^{-k} R\right)^{Q\left(\frac{1}{Q q}+\frac{1}{Q}-1\right)} \int_{\tilde{B}\left(\xi, c 2^{-k+1} R\right)} \sum_{i=1}^{m}\left|\tilde{X}_{i} \tilde{f}\right|(\eta) d \eta \\
& \leq C \sum_{k=0}^{\infty}\left(2^{-k} R\right)^{Q\left(\frac{1}{Q q}+\frac{1}{Q}-1\right)} \cdot K\left(2^{-k} R\right)^{Q-1} \\
& \leq C \sum_{k=0}^{\infty}\left(2^{-k}\right)^{\frac{1}{q}} \cdot K R^{\frac{1}{q}} \\
& \leq C \frac{1}{1-2^{-\frac{1}{q}}} \cdot K R^{\frac{1}{q}} \\
& \leq C q K R^{\frac{1}{q}}
\end{aligned}
$$

Therefore,

$$
\int_{\tilde{B}}\left|\tilde{f}(\xi)-\tilde{f}_{\tilde{B}}\right|^{q} d \xi \leq(C q)^{q} K^{q} R^{Q} .
$$

This inequality holds for all $q \geq 1$, thus we have shown that

$$
\int_{\tilde{B}} e^{\mu\left|\tilde{f}(\xi)-\tilde{f}_{\tilde{B}}\right|} d \xi \leq C K R^{Q}
$$

provided $\mu$ (independent of $\tilde{B}$ and $\tilde{f}$ ) is not too large. The above inequality says

$$
\frac{1}{|\tilde{B}|} \int_{\tilde{B}} e^{\mu\left|\tilde{f}(\xi)-\tilde{f}_{\tilde{B}}\right|} d \xi \leq C K
$$


For any given function $f$ and ball $B=B\left(x_{0}, r\right) \subset \Omega$, we define $\tilde{B}=$ $\tilde{B}\left(\left(x_{0}, 0\right), r\right) \subset \tilde{\Omega}, \tilde{f}(x, t)=f(x)$. By using the following fact proved in [NSW]

$$
\int_{R^{l}} \chi_{\tilde{B}}(y, t) d t \leq C \frac{|\tilde{B}|}{|B|}
$$

we will get from $(2.3)$

$$
\frac{1}{|B|} \int_{B} e^{\mu\left|f(x)-f_{B}\right|} d x \leq C K .
$$

\section{The Harnack inequalities}

We will establish in this section certain Harnack inequalities for weak solutions, subsolutions, and supersolutions of quasilinear second order subelliptic partial differential equations of the form (1.4) under the structural conditions (3.1) below on the equation (1.4).

We recall that $x=\left(x_{1}, \ldots, x_{n}\right), \eta=\left(\eta_{1}, \ldots, \eta_{m}\right)$ denote vectors in $R^{n}$ and $R^{m}$ respectively and $X u=\left(X_{1} u, \ldots, X_{m} u\right)$ and $A(x, u, \eta)=$ $\left(A_{1}(x, u, \eta), \ldots, A_{m}(x, u, \eta)\right)$ and $B(x, u, \eta)$ denote, respectively, vector and scalar measurable functions defined on $\Omega \times R \times R^{m}$, where $\Omega$ is a domain in $R^{n}$.

The structure of the equation (1.4) throughout this paper will be assumed to satisfy the following:

$$
\begin{aligned}
|A(x, u, \eta)| & \leq a_{0}|\eta|^{p-1}+\left(a_{1}(x)|u|\right)^{p-1}+\left(a_{3}(x)\right)^{p-1} \\
\eta \cdot A(x, u, \eta) & \geq|\eta|^{p}-\left(a_{2}(x)|u|\right)^{p}-\left(a_{4}(x)\right)^{p} \\
|B(x, u, \eta)| & \leq b_{0}|\eta|^{p}+b_{1}(x)|\eta|^{p-1}+\left(b_{2}(x)\right)^{p}|u|^{p-1}+\left(b_{3}(x)\right)^{p}
\end{aligned}
$$

where $p>1, a_{0}, b_{0}$ are constants, $a_{i}(x), b_{i}(x)$ are nonnegative measurable functions satisfying certain integrability properties which will be described below.

We now define the notion of solutions, subsolutions and supersolutions of the equations (1.4). A function $u(x)$ is said to be a weak solution (subsolution, or supersolution) of (1.4) in $\Omega$ if $u(x) \in W_{\mathrm{loc}}^{1, p}(\Omega)$ and

$$
\int_{\Omega}\{X \phi \cdot A(x, u, X u)-\phi B(x, u, X u)\} d x=0(\leq 0, \text { or } \geq 0)
$$

for all bounded $\phi(x) \in W_{0}^{1, p}(\Omega)$. 
We note here that if (3.2) holds for all $\phi(x) \geq 0, \phi(x) \in C_{0}^{1}(\Omega)$ and $a_{i}(x), b_{i}(x) \in L_{\mathrm{loc}}^{Q}(\Omega), u(x) \in L_{\mathrm{loc}}^{\infty}$, then a standard argument of approximation will show that it still holds for all $\phi(x)$ given in the definition.

We now let $\epsilon(\rho)$ be a smooth function defined for $\rho>0$ and such that $\epsilon(\rho) \rightarrow 0$ as $\rho \rightarrow 0$. We also define the space $L^{Q, \epsilon(\rho)}$ by

$$
L^{Q, \epsilon(\rho)}=\left\{u(x) \in L^{Q}(\Omega):\|u\|_{Q, \epsilon(\rho) ; \Omega}<\infty\right\},
$$

where

$$
\|u\|_{Q, \epsilon(\rho) ; \Omega}=\sup _{x_{0} \in \Omega, \rho>0} \epsilon(\rho)^{-1}\|u\|_{Q ; B_{\rho}\left(x_{0}\right) \bigcap \Omega} .
$$

We assume the functions $a_{i}(x), b_{j}(x)$ in the structure condition (3.1) in such space with certain $\epsilon(\rho)$. More precisely, we will assume when $p<Q$ that

$$
a_{i}(x), b_{j}(x) \in L^{Q, \rho^{\alpha}}(\Omega) \text { for some } \alpha>0, \quad i=2,4 ; j=1,2,3
$$

and

$$
a_{i}(x) \in L^{Q}(\Omega), \quad i=1,3,
$$

and we in this case set $B=B_{3 \rho}\left(x_{0}\right)$ and

$$
\begin{aligned}
\lambda & =\rho^{-1}\left\|a_{1}\right\|_{Q ; B} \bigcap_{\Omega}+\rho^{\alpha-1}\left\|a_{2}+b_{1}+b_{2}\right\|_{Q, \rho^{\alpha} ; B} \bigcap^{\Omega} \\
m(\rho) & =\left\|a_{3}\right\|_{Q, B} \bigcap \Omega \\
& +\rho^{\alpha}\left\|a_{4}\right\|_{Q, \rho^{\alpha} ; B} \bigcap+\left(\rho^{\alpha}\left\|b_{3}\right\|_{Q, \rho^{\alpha} ; B} \bigcap^{\frac{p}{p-1}}\right.
\end{aligned}
$$

When $p=Q$, we also assume $a_{1}(x), a_{3}(x) \in L^{Q, \rho^{\alpha}}(\Omega)$ and set for $B=$ $B_{3 \rho}\left(x_{0}\right)$

$$
\begin{aligned}
\lambda & =\rho^{\alpha-1}\left\|a_{1}+a_{2}+b_{1}+b_{2}\right\|_{Q, \rho^{\alpha} ; B} \bigcap \Omega \\
m(\rho) & =\rho^{\alpha}\left\|a_{3}+a_{4}\right\|_{Q, \rho^{\alpha} ; B} \bigcap_{\Omega}+\left(\rho^{\alpha}\left\|b_{3}\right\|_{Q, \rho^{\alpha} ; B} \bigcap \Omega\right)^{\frac{p}{p-1}} .
\end{aligned}
$$

If $p>Q$ we assume that all $a_{i}, b_{j}$ are in $L^{p}(\Omega)$ and set

$$
\begin{aligned}
& \lambda=\rho^{-Q / p}\left\|a_{1}+a_{2}+b_{1}+b_{2}\right\|_{Q, \rho^{\alpha} ; B} \bigcap \Omega \\
& m(\rho)=\rho^{1-Q / p}\left\|a_{3}+a_{4}\right\|_{Q, \rho^{\alpha} ; B} \bigcap \Omega \\
&+\left(\rho^{1-Q / p}\left\|b_{3}\right\|_{p, \rho^{\alpha} ; B} \bigcap \Omega\right)^{\frac{p}{p-1}} .
\end{aligned}
$$

Remark. If we only assume $\epsilon(\rho)>0$ satisfies a certain Dini condition, i.e., $\int_{0}^{1} \frac{\epsilon(\rho)}{\rho} d \rho<\infty$, then the proofs of all the theorems below still hold with minimal modifications.

Besides the embedding theorems proved in Section 1, we also need the following lemma to prove the Harnack inequality. 
Lemma 3.7. Suppose that $u(x) \in W_{0}^{1, p}(\Omega), f(x) \in L^{Q, \rho^{\alpha}}(\Omega)$ if $p<$ $Q ; f(x) \in L_{\mathrm{loc}}^{t}(\Omega)$ if $p=Q ; f(x) \in L^{p}(\Omega)$ if $p>Q$. Then for any $\epsilon>0$

$$
\|f u\|_{p, \Omega} \leq \epsilon|| X u\left\|_{p, \Omega}+C(p, Q, \alpha, \Omega,\|f\|) \epsilon^{-\beta}\right\| u \|_{p, \Omega},
$$

where $\beta=\beta(p, Q)>0$ if $p>Q$ and $\beta=\beta(p, Q, \alpha)>0$ if $p \leq Q$.

Remark. When $p<Q$, if we only assume $f \in L_{\mathrm{loc}}^{Q}(\Omega)$ but assume the $L^{Q}$ norm is small then this lemma still holds as one can see from the proof given below.

Proof: We first assume $p<Q$. Given each fixed small enough $r>0$. Then we can find a partition of unity of the domain $\Omega$. More precisely, there exists a finite sequence of metric balls $B_{i}=B\left(x_{i}, r\right), i=$ $1,2, \ldots, M$ and functions $\eta_{i}(x), i=1,2, \ldots, M$ such that $\operatorname{supp}\left\{\eta_{i}\right\} \subset$ $B_{i},\left|X \eta_{i}\right| \leq C r^{-1}, \Omega \subset \bigcup_{i=1}^{M} B_{i}$ and $\sum_{i=1}^{M} \eta_{i}^{p}(x)=1$ for all $x \in \Omega$. Thus if we set $u_{i}(x)=u(x) \eta_{i}(x)$ for $i=1, \ldots, M$

$$
\begin{aligned}
\int_{\Omega} f(x)^{p} u(x)^{p} d x & =\sum_{i=1}^{M} \int_{B_{i}} f^{p}(x) u^{p}(x) \eta_{i}^{p}(x) d x=\sum_{i=1}^{M} \int_{B_{i}} f^{p}(x) u_{i}^{p}(x) d x \\
& \leq \sum_{i=1}^{M}\left(\int_{B_{i}} f^{Q}(x) d x\right)^{p / Q} \cdot\left(\int_{B_{i}} u_{i}^{\frac{p Q}{Q-p}}(x) d x\right)^{\frac{Q-p}{Q}} \\
& \leq \sum_{i=1}^{M}\left(\int_{B_{i}} f^{Q}(x) d x\right)^{p / Q} \cdot\left(\int_{B_{i}}\left|X u_{i}\right|^{p}(x) d x\right) \\
& \leq C r^{\alpha p} \sum_{i=1}^{M}\left(\int_{B_{i}} \eta_{i}^{p}(x)|X u|^{p}(x) d x+\int_{B_{i}} u^{p}(x)\left|X \eta_{i}(x)\right|^{p} d x\right) \\
& \leq C r^{\alpha p} \int_{\Omega}|X u|^{p}+C r^{\alpha p-p} \int_{\Omega}|u|^{p} .
\end{aligned}
$$

We note that we have used the following Sobolev inequality since $u_{i}(x)$ has support in $B_{i}$ (see, for example, Theorem $\mathrm{C}$ in $[\mathbf{L 1}]$ )

$$
\left(\int_{B_{i}} u_{i}(x)^{\frac{p Q}{Q-p}} d x\right)^{\frac{Q-p}{Q p}} \leq C\left(\int_{B_{i}}\left|X u_{i}\right|^{p}\right)^{\frac{1}{p}}
$$

If we replace the constant $C r^{\alpha p}$ by $\epsilon>0$ we will get our proof. We note the precise constant $C(r, p, Q, \alpha)$ can be calculated. 
Now let $p>Q$, we use the same partition of unity as above. Then

$\int_{\Omega} f(x)^{p} u(x)^{p} d x=\sum_{i=1}^{M} \int_{B_{i}} f^{p}(x) u^{p}(x) \eta_{i}^{p}(x) d x=\sum_{i=1}^{M} \int_{B_{i}} f^{p}(x) u_{i}^{p}(x) d x$.

We note again $\operatorname{supp}\left\{u_{i}\right\} \subset B_{i}$ and $p>Q$, then we have by Theorem (1.1) in Section $1, u_{i}(x) \in L^{\infty}\left(B_{i}\right)$ and its norm is bounded by $C r^{1-Q / p}\left\|X u_{i}\right\|_{p, B_{i}} \leq C r^{1-Q / p}\left\|X u_{i}\right\|_{p, \Omega}$. Therefore,

$$
\begin{aligned}
\int_{\Omega} f(x)^{p} u(x)^{p} d x & \leq \sum_{i=1}^{M} \int_{B_{i}} f^{p}(x) \cdot r^{p-Q}\left\|X u_{i}\right\|_{p, \Omega}^{p} \\
& \leq C r^{p-Q} \cdot\|f\|_{p, \Omega}^{p} \cdot\|X u\|_{p, \Omega}^{p}+C \mid f\left\|_{p, \Omega} r^{-Q}\right\| u \|_{p, \Omega}
\end{aligned}
$$

Then by setting $\epsilon=C r^{p-Q} \cdot\|f\|_{p, \Omega}^{p}$ we will get the proof.

When $p=Q, u_{i}$ is exponentially integrable as shown in $[\mathbf{L 3}]$ and especially in $L_{\text {loc }}^{t}$ for all $t>Q$. We now assume $f \in L_{\text {loc }}^{t}(\Omega)$ for some $t>Q$, then arguing as above

$$
\begin{aligned}
\int_{\Omega} f(x)^{Q} u(x)^{Q} d x= & \sum_{i=1}^{M} \int_{B_{i}} f^{Q}(x) u^{Q}(x) \eta_{i}^{p}(x) d x=\sum_{i=1}^{M} \int_{B_{i}} f^{Q}(x) u_{i}^{Q}(x) d x \\
\leq & \sum_{i=1}^{M}\left(\int_{B_{i}} f^{t}(x) d x\right)^{Q / t} \cdot\left(\int_{B_{i}} u_{i}^{\frac{t Q}{t-Q}}(x) d x\right)^{\frac{t-Q}{t}} \\
\leq & \sum_{i=1}^{M}\left(\int_{B_{i}} f^{t}(x) d x\right)^{Q / t} \cdot r^{Q(t-Q) / t}\left(\int_{B_{i}}\left|X u_{i}\right|^{Q}(x) d x\right) \\
\leq & C r^{Q(t-Q) / t}|| f \|_{t, \Omega}^{Q} \sum_{i=1}^{M}\left(\int_{B_{i}} \eta_{i}^{Q}(x)|X u|^{Q}(x) d x\right. \\
& \left.+\int_{B_{i}} u^{Q}(x)\left|X \eta_{i}(x)\right|^{Q} d x\right) \\
\leq & C r^{Q(t-Q) / t}|| f \|_{t, \Omega}^{Q} \int_{\Omega}|X u|^{Q}+C(r, p, Q, t) \int_{\Omega}|u|^{Q} .
\end{aligned}
$$

Taking $C r^{\frac{Q(t-Q)}{t}}\|f\|_{t, \Omega}^{Q}=\epsilon$, we will get the desired result.

All the results proved in this paper will be of local nature. We will simply denote a ball of radius $\rho$ as $B_{\rho}$ and drop the center in the notation because the centers are not important here. 
Theorem 3.9. Suppose that $u(x)$ is a nonnegative weak solution of (1.4) in a metric ball $B_{3 \rho} \subset \Omega$ with $0 \leq u<M$ in $B_{3 \rho}$. Then

$$
\max _{B_{\rho}} u(x) \leq C\left(\min _{B_{\rho}} u(x)+m(\rho)\right)
$$

where $C=C\left(p, Q, a_{0}, b_{0} M, \lambda \rho\right)$.

For the standard Harnack inequality stated below to hold, we need to assume that $a_{3}(x), a_{4}(x), b_{3}(x)=0$.

Corollary 3.11. Suppose that $u(x)$ is a nonnegative weak solution of (1.4) in a metric ball $B_{3 \rho} \subset \Omega$ with $0 \leq u<M$ in $B_{3 \rho}$. Assume that $a_{3}(x), a_{4}(x), b_{3}(x)=0$. Then

$$
\max _{B_{\rho}} u(x) \leq C \min _{B_{\rho}} u(x)
$$

where $C=C\left(p, Q, a_{0}, b_{0} M, \lambda \rho\right)$.

The special case of our theorem, i.e., $b_{0}=0$ has been found in [CDG1] when $1<p \leq Q$, but with stronger assumptions on the coefficients $a_{i}(x)$ and $b_{j}(x)$. In this case $b_{0}=0$, we do not need to assume the boundedness of $u(x)$ provided that the functions in the structure conditions (3.1) do not depend on $M$ (since $\left.b_{0} M=0\right)$. We treat all the cases $1<p<\infty$ here in a unified way. One of the main features is the availability of the new embedding theorem proved in this paper.

For the weak supsolutions of (1.4) we have the following weak Harnack inequality.

Theorem 3.13. Suppose that $u(x)$ is a weak supsolution of (1.4) in a metric ball $B_{3 \rho} \subset \Omega$ with $0 \leq u<M$ in $B_{3 \rho}$. Then

$$
\rho^{\frac{-Q}{\gamma}}\|u(x)\|_{\gamma, B_{2 \rho}} \leq C\left(\min _{B_{\rho}} u(x)+m(\rho)\right)
$$

for any $\gamma<\frac{Q(p-1)}{Q-p}$ if $p \leq Q, \gamma \leq \infty$ if $p>Q$ and where $C=$ $C\left(p, Q, a_{0}, b_{0} M, \lambda \rho\right)$.

For the weak subsolutions of (1.4) we have the following estimate: 
Theorem 3.15. Suppose that $u(x)$ is a weak subsolution of (1.4) in a metric ball $B_{3 \rho} \subset \Omega$ with $0 \leq u<M$ in $B_{3 \rho}$. Then

$$
\max _{B_{\rho}} u(x) \leq C\left(\rho^{\frac{-Q}{\gamma}}\|u(x)\|_{\gamma, B_{2 \rho}}+m(\rho)\right)
$$

for any $\gamma>p-1$, where $C=C\left(p, Q, a_{0}, b_{0} M, \lambda \rho\right)$.

We remark here that Theorem (3.15) also holds for $p=1$ as one can see from the proof below. It is clear that Theorem (3.9) is a consequence of Theorems (3.13) and (3.15).

The proofs of the above theorems adapt the well-known iteration argument of Moser [Mos]. More closely related arguments can be found in $[\mathbf{S e r}],[\mathbf{G i T}],[\mathbf{T r u}]$ and citeZie. We now define the functional

$$
\phi(s, h)=\left\{\frac{1}{\left|B_{h}\right|} \int_{B_{h}}|u|^{s} d x\right\}^{\frac{1}{s}}, \quad s \neq 0, h>0 .
$$

Thus

$$
\begin{aligned}
\phi(\infty, \rho) & =\max _{B_{\rho}} u(x), \\
\phi(-\infty, \rho) & =\min _{B_{\rho}} u(x) .
\end{aligned}
$$

Consequently, the inequalities (3.10), (3.14) and (3.16) may be written as

$$
\begin{aligned}
& \phi(\infty, \rho) \leq C(\phi(-\infty, \rho)+m(\rho)), \\
& \phi(\gamma, 2 \rho) \leq C(\phi(-\infty, \rho)+m(\rho)), \\
& \phi(\infty, \rho) \leq C(\phi(\gamma, 2 \rho)+m(\rho)) .
\end{aligned}
$$

Before we prove all the Harnack inequalities we first make the following reductions. We define

$$
\begin{aligned}
\overline{a_{2}(x)} & =a_{2}(x)+m(\rho)^{-1} a_{4}(x) \\
\overline{b_{2}(x)} & =b_{2}(x)+m(\rho)^{\frac{1}{p}-1} b_{3}(x) \\
\overline{a_{1}(x)} & =a_{1}(x)+m(\rho)^{-1} a_{3}(x) \\
\overline{u(x)} & =u(x)+m(\rho) .
\end{aligned}
$$

Thus $\overline{u(x)}$ will satisfy an equation of the form (1.4)

$$
\sum_{j=1}^{m} X_{j}^{*} \overline{A_{j}}\left(x, \bar{u}, X_{1} \bar{u}, X_{2} \bar{u}, \ldots, X_{m} \bar{u}\right)+\bar{B}\left(x, \bar{u}, X_{1} \bar{u}, X_{2} \bar{u}, \ldots, X_{m} \bar{u}\right)=0
$$


where $\bar{A}(x, u, \eta)$ and $\bar{B}(x, u, \eta)$ satisfies the following conditions:

$$
\begin{aligned}
|\bar{A}(x, u, \eta)| & \leq a_{0}|\eta|^{p-1}+\left(\overline{a_{1}}(x)|u|\right)^{p-1} \\
\eta \cdot \bar{A}(x, u, \eta) & \geq|\eta|^{p}-\left(\overline{a_{2}}(x)|u|\right)^{p} \\
|\bar{B}(x, u, \eta)| & \leq b_{0}|\eta|^{p}+b_{1}(x)|\eta|^{p-1}+\left(\overline{b_{2}}(x)\right)^{p}|u|^{p-1} .
\end{aligned}
$$

Therefore this reduces the structure conditions to the cases $\overline{a_{3}(x)}=$ $\overline{b_{3}(x)}=\overline{a_{4}(x)}=0$, i.e., $m(\rho)=0$. For simplicity we will also drop the "bar" from $\bar{A}, \bar{B}, \overline{u(x)}, \overline{a_{1}}(x), \overline{a_{2}}(x), \overline{b_{2}}(x)$ and simply write $A, B, u(x)$, $a_{1}(x), a_{2}(x), b_{2}(x)$.

Proof of Theorem (3.9): We assume with no loss of generality that $u(x) \geq \epsilon>0$. We select a test function in (3.2)

$$
\phi(x)=\xi^{p}(x) u^{q}(x) e^{(\mathbf{s g n} \mathbf{q}) b_{0} u(x)},
$$

where $q \neq 0$ and $\xi(x) \geq 0, \xi(x) \in C_{0}^{\infty}\left(B_{3 \rho}\right)$ will be specified later. By (3.21), we have

$X \phi(x)=($ sgn q $) \xi^{p} e^{(\text {sgn q }) b_{0} u}\left(b_{0} u^{q}+|q| u^{q-1}\right) X u+p \xi^{p-1} u^{q} e^{(\text {sgn q }) b_{0} u} X \xi$, where $X f=\left(X_{1} f, \ldots, X_{m} f\right)$ is the subelliptic gradient vector for the given function $f$. Substituting (3.21) and (3.22) into (3.2) we get

$$
\begin{aligned}
& \text { (sgn q) } \int_{B} \xi^{p} e^{\left(\text {sgn q) } b_{0} u\right.}\left(b_{0} u^{q}+|q| u^{q-1}\right) X u \cdot A(x, u, X u) \\
& +p \int_{B} \xi^{p-1} u^{q} e^{(\mathbf{s g n} \mathbf{q}) b_{0} u} X \xi \cdot A(x, u, X u)-\int_{B} \xi^{q} e^{(\mathbf{s g n} \mathbf{q}) b_{0} u} u^{q} B(x, u, X u) \\
& \leq 0 \text { if } u \text { is a subsolution, ( } \geq 0 \text { if } u \text { is a supersolution.) }
\end{aligned}
$$

The above $\cdot$ stands for the inner product.

In the following calculations, it will be understood that $q>0$ when $u$ satisfies the hypothesis of Theorem (3.15) and that $q<0$ when $u$ satisfies the hypothesis of Theorem (3.13).

By employing the structure condition (3.20) and together with (3.23), we get

$$
\begin{aligned}
& \int_{B_{3 \rho}} e^{(\text {sgn q }) b_{0} u} \xi^{p}\left(b_{0} u^{q}+|q| u^{q-1}\right)|X u|^{p} \\
& \quad \leq \int_{B_{3 \rho}} e^{(\text {sgn q }) b_{0} u} \xi^{p}\left(b_{0} u+|q|\right) a_{2}^{p} u^{p+q-1} \\
& \quad+p \int_{B_{3 \rho}} e^{(\text {sgn q }) b_{0} u} \xi^{p-1}|X \xi|\left(a_{0}|X u|^{p-1}+a_{1}^{p-1} u^{p-1}\right) u^{q} \\
& \quad+\int_{B_{3 \rho}} e^{\left(\text {sgn q) } b_{0} u\right.} \xi^{p}\left(b_{0}|X u|^{p}+b_{1}|X u|^{p-1}+b_{2}^{p} u^{p-1}\right) u^{q} .
\end{aligned}
$$


We note the term

$$
b_{0} \int_{B_{3 \rho}} e^{(\mathbf{s g n} \mathbf{q}) b_{0} u} \xi^{p} u^{q}|X u|^{p}
$$

can be dropped from both sides of (3.24).

After calculation and Hölder's inequality and together with the estimate $0 \leq b_{0} u<b_{0} M$, we can bootstrap the terms involving $|X u|$ and we will get

$$
\begin{aligned}
& \quad \int_{B_{3 \rho}} \xi^{p} u^{q-1}|X u|^{p} \\
& \leq C\left(1+|q|^{-1}\right)^{p} \int_{B_{3 \rho}}\left\{\left(\epsilon a_{1}+a_{2}+b_{1}+b_{2}\right)^{p} \xi^{p}+\epsilon^{\frac{p}{1-p}}|X \xi|^{p}\right\} u^{p+q-1}
\end{aligned}
$$

for any given $0<\epsilon \leq 1$.

Set $f(x)=\left(\epsilon a_{1}+a_{2}+b_{1}+b_{2}\right)$, then by Lemma (3.7) ( $\xi$ plays the role of $u$ there) and the assumptions on $a_{i}(x)$ and $b_{j}(x)(i, j=1,2)$ we get

$$
\int_{B_{3 \rho}} \xi^{p} u^{q-1}|X u|^{p} \leq C\left(1+|q|^{-1}\right)^{p} \int_{B_{3 \rho}}\left(\xi^{p}+|X \xi|^{p}\right) u^{p+q-1}
$$

where $C$ depends on $\lambda \rho$ (see definition of $\lambda$ at the beginning of this section) and etc.

We now let

$$
v(x)= \begin{cases}u^{t}(x) & \text { where } p t=p+q-1 \text { for } q \neq 1-p \\ \log u(x) & \text { for } q=1-p\end{cases}
$$

Thus (3.26) can be written as

$$
\|\xi X v\|_{p, B_{3 \rho}} \leq \begin{cases}C|t|\left(1+|q|^{-1}\right)\|(\xi+|X \xi|) v\|_{p, B_{3 \rho} \rho} & \text { for } q \neq 1-p, 0 \\ C|| \xi+\mid X \xi \|_{p, B_{3 \rho}} & \text { for } q=1-p .\end{cases}
$$

We consider the case $q \neq 1-p$ in (3.28). By Sobolev embedding lemma (Theorem $\mathrm{C}$ in $[\mathbf{L} \mathbf{1}]$ ), and the exponential integrability when $p=Q$ and Theorem (1.1) when $p>Q$ in Section 1, we get

$$
\|\xi v\|_{\chi p, B_{3 \rho}} \leq C|t|\left(1+|q|^{-1}\right) \rho\left|B_{3 \rho}\right|^{\frac{1}{\chi p}-\frac{1}{p}}\|(\xi+|X \xi|) v\|_{p, B_{3 \rho}}
$$

where $\chi=\frac{Q}{Q-p}$ if $p<Q$, and $\chi$ can be arbitarily large if $p=Q$, and $\chi=\infty$ if $p>Q$. Let now $r_{1}, r_{2}$ satisfy $\rho \leq r_{1}, r_{2} \leq 2 \rho$ and select $\xi(x)$ 
as a cut-off function such that $\xi(x)=1$ on $B_{r_{1}}$ and $\xi(x)=0$ outside $B_{r_{2}}$ and $|\xi(x)| \leq C\left(r_{2}-r_{1}\right)^{-1}$. The existence of such a cut-off function was proved in $[\mathbf{L} \mathbf{1}]$.

$$
\|v\|_{\chi p, B_{r_{1}}} \leq C|t|\left(1+|q|^{-1}\right)\left(r_{2}-r_{1}\right)^{-1} \rho\left|B_{3 \rho}\right|^{\frac{1}{\chi p}-\frac{1}{p}}\|v\|_{p, B_{r_{2}}} .
$$

We note here that $r_{1}, r_{2}, \rho$ are comparable, and also note that the Lebesgue measure is doubling with respect to the metric balls by the work of $[\mathbf{N S W}]$. Thus by taking $t$-th root of both sides of (3.30) and setting $s=p t=p+q-1$, we will get the following for positive $s$

$$
\phi\left(\chi s, r_{1}\right) \leq\left[C|t|\left(1+|q|^{-1}\right)\left(r_{2}-r_{1}\right)^{-1}\right]^{p / s} \phi\left(s, r_{2}\right),
$$

while for negative $s$ we get

$$
\phi\left(\chi s, r_{1}\right) \geq\left[C|t|\left(1+|q|^{-1}\right)\left(r_{2}-r_{1}\right)^{-1}\right]^{p / s} \phi\left(s, r_{2}\right) .
$$

We now fix some $s_{0}>0$ and define

$$
s=s_{j}=\chi^{j} s_{0}, r_{j}=\left(1+2^{-j}\right) \quad \rho, j=0,1,2, \ldots
$$

We assume $s_{0}$ is so selected that no $s_{j}$ will coincide with $p-1$ for otherwise $s=s_{j}=p-1$ and $q=0$. Therefore $1+|q|^{-1}<C$ for all $j$.

By (3.31) we obtain

$$
\begin{aligned}
\phi\left(s_{j+1}, r_{j+1}\right) & \leq\left[C(2 \chi)^{j}\right]^{\frac{p \chi^{-j}}{s_{0}}} \phi\left(s_{j}, r_{j}\right) \\
& \leq C^{\sum \chi^{-j}}\left[C(2 \chi)^{p / s_{0}}\right]^{\sum j \chi^{-j}} \phi\left(s_{0}, 2 \rho\right) \leq C \phi\left(s_{0}, 2 \rho\right) .
\end{aligned}
$$

We have used the fact that $\chi>1$ and then the corresponding series in the above converges.

If we let $j \rightarrow \infty$ we will get

$$
\phi(\infty, \rho) \leq C \phi\left(s_{0}, 2 \rho\right)
$$

It is clear then for any $s_{0}=\gamma>p-1,(3.34)$ holds and then we have shown Theorem (3.15). Actually, Theorem (3.15) also holds when $p=1$ because in the above proof $s_{0}$ is allowed to be any positive number.

Suppose now that $u(x)$ is a supersolution, (3.33) holds for any $s_{0}>0$ and $s_{j}<p-1$ and thus

$$
\phi(\gamma, 2 \rho) \leq C \phi\left(s_{0}, \frac{5 \rho}{2}\right)
$$


for any $s_{0}>0, \gamma<\frac{Q(p-1)}{Q-p}$ if $p \leq Q$ and $\gamma \leq \infty$ if $p>Q$.

We note that the iteration of (3.32) will lead to

$$
\phi\left(-s_{0}, \frac{5 \rho}{2}\right) \leq C \phi(-\infty, \rho)
$$

for any $s_{0}>0$.

Therefore, if we can show that there exists some $s_{0}>0$ such that

$$
\phi\left(s_{0}, \frac{5 \rho}{2}\right) \leq C \phi\left(-s_{0}, \frac{5 \rho}{2}\right)
$$

then we will have proved Theorem (3.13).

We now let $B_{r}$ be any ball contained in $B_{\rho_{0}}$ and choose $\xi(x)$ such that $\xi(x)=1$ on $B_{r}$ and 0 outside $B_{2 r}$ and $|\xi(x)| \leq C r^{-1}$. Then we get

$$
\|X v\|_{p, B_{r}} \leq C r^{\frac{Q-p}{p}}
$$

where $v$ is as in (3.28) when $q=p-1$. Thus Theorem (3.9) and (3.13) will follow from Theorem (1.3) in Section 1.

One application of the above theorem is the Hölder continuity of the weak solutions of (1.4).

Theorem 3.37. Suppose that $u(x)$ is a weak solution of (1.4) in $\Omega$ which is also locally bounded. Then $u(x)$ is Hölder continuous in $\Omega$ and if $B_{\rho_{0}} \subset \Omega$ then

$$
\operatorname{osc}_{B_{\rho}} u(x) \leq C\left(\frac{\rho}{\rho_{0}}\right)^{\alpha}\left\{\sup _{B_{\rho_{0}}}|u(x)|+m\left(\rho_{0}\right)\right\},
$$

for all $B_{\rho} \subset B_{\rho_{0}}$ and some $\alpha>0$, and $C=C\left(p, Q, a_{0}, b_{0} M\right)$.

The proof of the above theorem is fairly standard and we omit the details.

We now consider the estimates of the solutions at the boundary of the certain domains.

Let $S$ be a subset of $\partial \Omega$ and $u(x) \in W_{\text {loc }}^{1, p}(\Omega)$. Then we say that $u \leq D$ on $S$ if for every $\epsilon>0$ there is a neighborhood of $S$, called $\mathcal{M}_{S}$, such that $u \leq D+\epsilon$ a.e. in $\Omega \cap \mathcal{M}_{S}$. With such a definition we may easily define the notions $\sup _{S} u, \inf _{S} u$ and $\operatorname{ssc}_{S} u=\sup _{S} u-\inf _{S} u$. 
We consider the equation

$(3.39)$

$\sum_{j=1}^{m} X_{j}^{*} A_{j}\left(x, u, X_{1} u, X_{2} u, \ldots, X_{m} u\right)+B\left(x, u, X_{1} u, X_{2} u, \ldots, X_{m} u\right)=0$

under the following structure condition (for simplicity):

$$
\begin{aligned}
|A(x, y, \eta)| & \leq a_{0}|\eta|^{p-1}+a_{3} \\
\eta \cdot A(x, u, \eta) & \geq|\eta|^{p}-a_{4} \\
|B(x, u, \eta)| & \leq b_{0}|\eta|^{p}-b_{3}^{p} .
\end{aligned}
$$

Then

Theorem 3.41. Let $u(x)$ be a weak solution of (3.39) in $\Omega$. Let $B=B_{3 \rho}\left(x_{0}\right)$ and

$$
\begin{gathered}
L=\sup _{B \bigcap \partial \Omega} u(x), \\
M=\sup _{B \bigcap \Omega} u(x) .
\end{gathered}
$$

Then the function $v(x)$ given by

$$
v(x)= \begin{cases}M-\sup (u, L) & \text { for } x \in \Omega \bigcap B \\ M-L & \text { for } x \in B \backslash \Omega\end{cases}
$$

will satisfy

$$
\rho^{-\frac{Q}{p-1}}\|v\|_{p-1 ; B_{2 \rho}} \leq C\left\{\min _{B_{\rho}} v+m(\rho)\right\}
$$

where $C=C\left(p, Q, a_{0}, b_{0}, M\right)$.

We now introduce the notion of a "regular point" on the boundary $\partial \Omega$. A point $x_{0} \in \partial \Omega$ is called "regular" if there exists a positive constant $\rho_{0}=\rho\left(x_{0}\right)$ such that for all $\rho \leq r_{0}$

$$
\left|B_{\rho}\left(x_{0}\right) \backslash \Omega\right| \geq \theta_{0}\left|B_{\rho}\left(x_{0}\right)\right| \text {. }
$$

If every point of $\partial \Omega$ is regular we say $\partial \Omega$ is regular, and it is called "uniformly regular" if $\rho_{0}$ and $\theta_{0}$ can be selected independent of $x_{0}$. 
Corollary 3.44. Let $u(x)$ satisfy the hypotheses of Theorem (3.41) and suppose that $x_{0} \in \partial \Omega$ is regular. Then for all $\rho \leq \rho_{0}$,

$$
\sup _{B_{\rho}} u(x)-L \leq \epsilon_{0}(M-L)+C m(\rho),
$$

where $\epsilon_{0}<1$ and $C$ is a positive constant depending on $p, Q, a_{0}, b_{0}, M$ and $\theta_{0}$.

We also state a theorem which is an extension of Theorem (3.37) to the boundary.

Theorem 3.46. Suppose that $u(x)$ is a weak solution of (3.39) in $\Omega$ which is also locally bounded. Let $x_{0} \in \Omega$ be regular. Then for any $\rho \leq \rho_{0}, \gamma<1$,

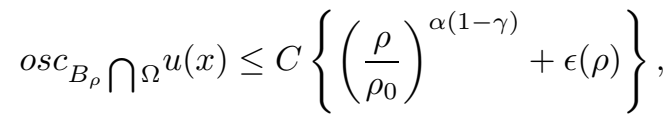

where $C$ and $\alpha>0$ depend on $p, Q, a_{0}, b_{0}, \sup _{B_{\rho_{0}}} u, \theta_{0}$ and $\rho_{*}$ and $\epsilon(\rho)=o s c_{\partial \Omega} \bigcap B_{\rho^{\gamma} \rho_{0}^{1-\gamma}} u$.

All the proofs of the above Theorems (3.41)-(3.46) follow by modifying the proofs of corresponding Theorems (3.9)-(3.15) and (3.37) and we omit the details. One needs to assume that all the vector fields are well defined and satisfying the Hörmander's condition in a larger domain $\Omega_{1}$ containing $\bar{\Omega}$ so that all the embedding theorems hold for those balls considered in the theorems.

After the paper was written and first circulated (with a slightly longer title) in February 1994, we learnt that some related work on Poincare estimates has also been obtained in $[\mathbf{B M}],[\mathbf{M S}],[\mathbf{C o u} 2],[\mathbf{H K}]$. A Poincaré type inequality with $\left|f(x)-f_{B}\right|$ replaced by $\left|f(x)-f\left(x_{0}\right)\right|$ for solutions to subelliptic quasilinear equations studied in the current paper has been given in [L5] for $p \geq 1$ and in [BKL] for $p<1$, among other things. We also became aware of the work $[\mathbf{H H}]$ for Harnack estimates on Carnot groups in conjunction with the quasiregular mappings, and the interior regularity for subelliptic systems $[\mathbf{X Z}]$, and isoperimetric inequality independently derived in $[\mathbf{C D G} 2]$ similar to that in $[\mathbf{F G W}]$.

Acknowledgement. The author wishes to thank the referee for his many useful comments and remarks, which help and improve the exposition of the paper. 


\section{References}

[BM] M. Biroli And U. Mosco, Proceedings of the Conference: Potential theory and partial differential operators with nonnegative characteristic form, Parma, February 1994, Kluwer, Amsterdam, to appear.

[Bon] J.-M. Bony, Principe du maximum, inégalité de Harnack et unicité du probleme de Cauchy pour les opérateurs elliptiques dégénérés, Ann. Inst. Fourier (Grenoble) 19 (1969), 277-304.

[BKL] S. Buckley, P. Koskela And G. Lu, Subelliptic Poincaré inequalities: the case $p<1$, Publ. Mat. 39 (1995), 313-334.

[CDG1] L. Capogna, D. Danielli and N. Garofalo, A embedding theorem and the Harnack inequality for solutions of nonlinear subelliptic equations, Comm. Partial Differential Equations 18(9-10) (1993), 1765-1794.

[CDG2] L. Capogna, D. Danielli and N. Garofalo, An isoperimetric inequality and the geometric Sobolev embedding for vector fields, Comm. Anal. Geom. 2(2) (1994), 203-215.

[Cou1] T. Coulhon, Inegalites de Gagliardo-Nirenberg pour les semi-groupes d'operateurs et applications, Potential Anal. 1 (1992), 343-353.

[Cou2] T. Coulhon, Espaces de Lipschitz et inegalites de Poincaré, J. Funct. Anal., to appear.

[FeP] C. FefFerman And D. H. Phong, Subelliptic eigenvalue problems, in "Proc. Conf. on Harmonic analysis," in honor of A. Zygmund, Wadsworth Math. Series, 1981, pp. 590-606.

[FeS] C. Fefferman and A. Sanchez-Calle, Fundamental solutions for second order subelliptic operators, Ann. of Math. 124(2) (1986), 247-272.

[Fr] B. Franchi, Weighted Sobolev-Poincaré inequalities and pointwise estimates for a class of degenerate elliptic operators, Trans. Amer. Math. Soc. 327 (1991), 125-158.

[FrL] B. FRANCHI AND E. LANCONELLI, Hölder regularity for a class of linear non uniformly elliptic operators, Ann. Scuola Norm. Sup. Pisa Cl. Sci. 10(IV) (1983), 523-541.

[FrS] B. Franchi And R. Serapioni, Pointwise estimates for a class of strongly degenerate elliptic operators, Ann. Scuola Norm. Sup. Pisa Cl. Sci. 14(IV) (1987), 527-568.

[FGW] B. Franchi, S. Gallot and R. L. Wheeden, Sobolev and isoperimetric inequalities for degenerate metrics, Math. Ann. 300 
(1994), 557-571.

[FGuW] B. Franchi, C. E. Gutierrez and R. L. Wheeden, Weighted Sobolev-Poincaré inequalities for Grushin type operators, Comm. Partial Differential Equations 19 (1994), 523-604.

[FLW] B. Franchi, G. Lu And R. L. Wheeden, Representation formula and weighted Poincaré inequalities for Hörmander vector fields, Ann. Inst. Fourier (Grenoble) 45(2), 577-604.

[Fol] G. B. Folland, Subelliptic estimates and function spaces on nilpotent Lie groups, Ark. Mat. 13 (1975), 161-207.

[FS] G. B. Folland And E. M. Stein, "Hardy spaces on homogeneous groups," Princeton Univ. Press, 1982.

[GiT] D. Gilbarg And N. Trudinger, "Elliptic partial differential equations of second order," Springer-Verlag, New York, 1983.

[HH] J. HeInONEN AND I. Holopainen, Quasiregular maps on Carnot groups, Preprint (1994).

[HK] P. Hajasz and P. Koskela, Sobolev meets Poincaré, C. $R$. Acad. Sci. Paris Sér. I Math. 320 (1995), 1211-1215.

[Hor] L. Hörmander, Hypoelliptic second order differential equations, Acta Math. 119 (1967), 147-171.

[Jer] D. JERISOn, The Poincaré inequality for vector fields satisfying Hörmander's condition, Duke Math. J. 53(2) (1986), 503-523.

[JeS] D. Jerison AND A. SANCHEz-CAlle, "Subelliptic second order differential operators," Complex Analysis, Lecture Notes in Mathematics 1277, Springer-Verlag, 1988.

[Kra] S. Krantz, Lipschitz spaces on stratified groups, Trans. Amer. Math. Soc. 269(1) (1982), 39-66.

[LaU] O. A. LAdyzhenskaya And N. N. URAL'tseva, On Holder continuity of solutions and their derivatives of linear and quasilinear elliptic and parabolic equations, in Russian, Trudy Mat. Inst. Steklov 73 (1964), 177-220.

[L1] G. Lu, Weighted Poincaré and Sobolev inequalities for vector fields satisfying Hörmander's condition and applications, Rev. Mat. Iberoamericana 8(3) (1992), 367-439.

[L2] G. Lu, The sharp Poincaré inequality for free vector fields: An endpoint result, Rev. Mat. Iberoamericana 10(2) (1994), 453-466. 
[L3] G. LU, Embedding theorems on Campanato-Morrey spaces for degenerate vector fields and applications, C. R. Acad. Sci. Paris Sér. I Math. 320 (1995), 429-434.

[L4] G. Lu, Existence and size estimates for the Green's functions of differential operators constructed from degenerate vector fields, Comm. Partial Differential Equations 17(7-8) (1992), 1213-1251.

[L5] G. Lu, A note on a Poincaré type inequality for solutions to subelliptic equations, Comm. Partial Differential Equations 21(1-2) (1996), 235-254.

[MSc] P. Maheux and L. Saloff-Coste, Analyse sur les boules d'un operateur sous-elliptique, Math. Ann., to appear.

[Mos] J. Moser, On Harnack's theorem for elliptic differential equations, Comm. Pure Appl. Math. 14 (1961), 577-591.

[NSW] A. NAGel, E. M. Stein And S. Wainger, Balls and metrics defined by vector fields I, Basic properties, Acta Math. 155 (1985), 103-147.

[RoS] L. P. Rothschild and E. M. Stein, Hypoelliptic differential operators and nilpotent groups, Acta Math. 137 (1976), 247-320.

[San] A. SÁnchez-CALLE, Fundamental solutions and geometry of the sum of squares of vector fields, Invent. Math. 78 (1984), 143-160.

[SC] L. Saloff-Coste, Theoremes de Sobolev et inegalites de Trudinger sur certains groupes de Lie, C. R. Acad. Sci. Paris Sér. I Math. 306 (1988), 305-308.

[Ser] J. SERRIN, Local behaviour of solutions of quasilinear equations, Acta Math. 111 (1964), 302-347.

[Tru] N. TRUDINGER, On Harnack type inequalities and their applications to quasilinear elliptic equations, Comm. Pure and Appl. Math. XX (1967), 721-747.

[Va] N. VARopoulos, Fonctions harmoniques sur les groupes de Lie, C. R. Acad. Sci. Paris Sér. I Math. 304 (1987), 519-521.

[VS-CC] N. Varopoulos, L. Saloff-Coste and T. Coulhon, "Analysis and Geometry on Groups," Cambridge University Press, Cambridge, 1992.

[X1] C. J. XU, Regularity for quasilinear second order subelliptic equations, Comm. Pure Appl. Math. 45(1) (1992), 77-96.

[XZ] C. J. Xu AND C. ZuILY, Higher interior regularity for quasilinear subelliptic systems, Prepublications, Universite de Paris-Sud, Mathematiques, Preprint (1995). 
[Zie] W. ZiEMER, Mean values of subsolutions of elliptic and parabolic equations, Trans. Amer. Math. Soc. 279 (1983), 555-568.

Keywords. Embedding theorems, Harnack's inequality, Quasilinear subelliptic equations, sub-Laplacian, Hörmander's vector fields, Lipschitz and BMO spaces.

1991 Mathematics subject classifications: 46E35 and 35B65

\author{
Department of Mathematics \\ Wright State University \\ Dayton, Ohio 45435 \\ U.S.A. \\ e-mail: gzlu@discover.wright.edu
}

Primera versió rebuda el 31 de Juliol de 1995,

darrera versió rebuda el 29 de Novembre de 1995 